\title{
Stability Constraints of Markov State Kinetic Models Based on Routh- Hurwitz Criterion
}

\author{
Sarmis $\mathbf{M}^{1}$, Orjuela $\mathbf{R}^{2}$, Bouteiller $\mathrm{JC}^{1,3}$, Ambert $\mathbf{N}^{1}$, Legendre $\mathrm{A}^{1,2}$, Bischoff $\mathrm{S}^{1}$, Haeberlé $\mathrm{O}^{2}$ and Baudry $\mathbf{M}^{1,4 *}$ \\ ${ }^{1}$ Rhenovia Pharma SA, Mulhouse, France \\ ${ }^{2}$ MIPS Laboratory, Université de Haute-Alsace, rue des Frères Lumières, Mulhouse, France \\ ${ }^{3}$ Department of Biomedical Engineering, University of Southern California, Los Angeles, CA, USA \\ ${ }^{4}$ Western University of Health Sciences, Pomona, CA, USA
}

\begin{abstract}
In computational neuroscience, receptors, channels and more generally signaling pathways are often modeled with Markov state models to represent biochemical reactions, which are then implemented with bilinear equations. One of the goals of these models, once calibrated with experimental results is to predict the dynamics of the biological system they represent in response to molecular perturbations and therefore facilitate and enhance the success rate of drug discovery and development. To model receptors under both pathological and physiological conditions, modelers usually modify the ligand association and dissociation parameters in the kinetic model during the optimization phase of model development. However, some parameter values may lead to unstable models, making calibration very difficult, time-consuming and inefficient before performing predictive in silico studies. In order to guarantee model stability during the parameter optimization phase, we propose to linearize bilinear kinetic models around an operating point. Considering the model input as piecewise constant, we propose an algorithm based on the Routh-Hurwitz criterion to generate stability constraints on model parameters. As an example, we apply this algorithm to the gamma-aminobutyric acid (GABA) receptor subtype A (GABAA receptor) model, as developed by Pugh and Raman (2005). The results obtained with the Routh-Hurwitz criterion provide constraint equations. These equations, once integrated into the parameter optimization process, guarantee the stability of the model and thus the success of the optimization process. An additional benefit is that the constraint equations allow determining the boundaries of the stability domain of the model. In the example provided, the Routh-Hurwitz criterion indicates that the model with the chosen parameters becomes unstable if GABA concentration rises above $6.54 \mathrm{mM}$. The proposed algorithm has also the advantage of being fast and easy to implement.
\end{abstract}

Keywords: Stability constraints; Bilinear; Routh-Hurwitz; Kinetic model; Optimization

Abbreviations: CNS: Central Nervous System; SBML: System Biology Markup Language; RH: Routh-Hurwitz; GABA: GammaAminobutyric Acid; GABAA: Gamma-Aminobutyric Acid Subtype A; VDCC: Voltage-Dependent Calcium Channels

\section{Introduction}

One of the main goals of systems biology consists in providing an integrative description of living systems and organisms by using simulation of complex and interconnected mathematical models of metabolic networks and signaling pathways [1,2]. System Biology Markup Language (SBML) [3] constitutes a standard for modeling systems biology and uses an XML-based language. SBML inherently allows developing synaptic receptor models at the molecular level using kinetic parameters as well as integrating a variety of mechanisms, which modulate the physical and functional properties of receptors (i.e., rise time, decay time, amplitude, etc.). Numerical tools are used to simulate these synaptic receptor models.

Rhenovia Pharma has developed a simulation platform $\left(\right.$ RHENOMS $\left.^{\mathrm{st}}\right)$ for hippocampal glutamatergic synapses and its integration into complex neuronal networks by combining kinetic receptor models and models of other reactions taking place within synapses [4]. This platform has been used to perform in silico experiments [5] to better understand the dynamics of synaptic transmission under physiological and pathological conditions. In order to calibrate these models, it is necessary to optimize the model parameters such that simulated results reproduce selected experimental results. In this optimization phase, the modeler iteratively modifies model parameters until simulated results match experimental ones
(Figure 1). In addition, in order to perform in silico simulations under normal and pathological conditions to test the effects of drugs for neurodegenerative or other central nervous system (CNS) diseases, both 'physiological' and 'pathological' synaptic receptor models have to be developed. To characterize the behavior of those 'control' and 'diseaselike' models, users go through an initial parameter optimization phase, which is very important to ensure that the model displays the desired behavior under both control and pathological conditions. However, the chosen parameter values may lead to an unstable model. To address this issue, stability conditions could need to be investigated from the mathematical structure of the kinetic model.

From a modeling point of view, biological kinetic models [6-8], particularly synaptic receptor models [9-11], represent states and transition probabilities between the different states (i.e., open or desensitized probability states). The resulting model, called Markov kinetic model, is often affine in control, which is a particular form of nonlinearity. These kinds of models are known as bilinear systems and

${ }^{*}$ Corresponding author: Michel Baudry, Western University of Health Sciences, Pomona, CA, USA, Tel: (+33) 389321 180; Fax: (+33) 389555 145; E-mail: mbaudry@westernu.edu

Received August 31, 2015; Accepted September 21, 2015; Published September 26, 2015

Citation: Sarmis M, Orjuela R, Bouteiller JC, Ambert N, Legendre A, et al. (2015) Stability Constraints of Markov State Kinetic Models Based on Routh-Hurwitz Criterion. J Comput Sci Syst Biol 8: 296-303. doi:10.4172/jcsb.1000204

Copyright: ( 2015 Sarmis M, et al. This is an open-access article distributed under the terms of the Creative Commons Attribution License, which permits unrestricted use, distribution, and reproduction in any medium, provided the original author and source are credited. 


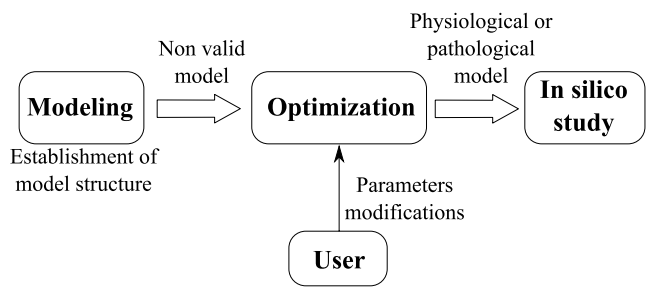

Development of a model generally requires three steps. The first step, the modeling, generates an initial, not-validated model. To obtain a physiologic or pathologic model, users modify the model parameters during the optimization step. The optimization step is run until desired physiological or pathological model is reached. The third step, or the study step, represents the application stage of the model, when new predictions can be generated by the model.

Figure 1: Schematic representation of the modeling and simulation process.

are commonly modeled by an Ordinary Differential Equations (ODE) [12-14] as follows:

$$
\dot{x}(t)=A x(t) \sum_{i=1}^{m} B_{i} u_{i}(t) x(t)
$$

where $u(t) \in \mathbb{R}^{n}$ is the system state, $u(t) \in \mathbb{R}^{n}$ are the external inputs with $u_{i}(t) \in \mathbb{R}$ the $i^{\text {th }}$ input $\left.i=1,2, \ldots, \mathrm{m}\right)$ and $B_{i} \in \mathbb{R}^{n \times m}$ its associated real constant matrix, and $A \in \mathbb{R}^{n \times n}$ is a real constant matrix. Notice that the analytical solution of this system cannot be often obtained due to the complexity and nonlinearities of the system. However under some conditions, stability conditions are proposed, as mentioned by Chen et al. [15]. Most studies consider time-invariant continuous bilinear systems with linear feedback [16-18]. Almost all of these suggest studying stability by finding a sufficient condition for the existence of a feedback control, such that the resulting closed-loop system is asymptotically stable.

A large number of publications study the stability of bilinear systems with the Lyapunov functions $[19,20]$. This energy based method developed by Lyapunov in 1892 [21], consists in finding sufficient conditions for stability of nonlinear systems. The sufficient conditions require the existence of Lyapunov functions $u(t)$ for the system or the existence of some negative (or positive) definite matrices, such that the feedback input $u(t)$ depends on those matrices as shown in ref. $[22,23]$. This method avoids the explicit solution of the ODE and can then be applied to a large class of nonlinear systems. This method could represent an interesting way to solve the problem investigated in this paper. However, for each modification of a given parameter, another function $V(x)$ needs to be determined during the optimization phase, increasing the computation-time. In order to reduce the mathematical complexity of the investigated model, an approximation (i.e., a linearization) around an operating point can be proposed considering the system input $u(t)$ as a piecewise constant signal. In this way the nonlinear model is transformed into a linear system and some well-known stability conditions can be easily employed based on the computation of the eigenvalues of the dynamic matrix.

Among them, Routh-Hurwitz (RH) stability criterion is a mathematical test, which provides a necessary and sufficient condition to characterize the stability of a linear system without computing explicitly the solution of the eigenvalues (roots), thus reducing computing time. This test is well adapted to biological bilinear systems, which are considered here because they may become extremely complex, consequently making it difficult (computationally speaking) and sometimes even impossible to compute the eigenvalues (roots). In addition, it is also important to note that even if the proof of stability is provided for the operating point no constraints are available to guarantee the stability during the optimization step.

Studying the kinetic model to find the proof of stability is not enough in this presented model tuning process. During the model tuning process, users modify the model associations and dissociations parameters (with an optimization algorithm). The problematic encountered is that some parameter values and simulation conditions may lead to model instability resulting in (i) lack of convergence of the simulation runs, (ii) erroneous solutions of the sets of ODE used to solve the model equations, and (iii) prohibitively long simulation durations. This instability may jeopardize model simulation and consequently hinder the success of the parameter optimization phase. These issues led us to ask whether it could be possible to identify constraints on parameter values, which could guarantee model stability. Finding these stability constraints is the primary objective of this study in order to guarantee the optimization step success.

In this manuscript, the stability concept and its application for linear and bilinear models are presented in Section 2. Then, the proposed approach based on the Routh-Hurwitz stability criterion is presented. The RH criterion efficacy is demonstrated with two examples, a textbook model and the GABA receptor model. Finally, discussion about the results obtained with the $\mathrm{RH}$ criterion as well as their limitations and elaborates on the perspectives of this work.

\section{Model Stability}

To represent the notions of stability and equilibrium point one must first define the system of interest. Let's consider a process represented by the following nonlinear equation:

$$
\dot{x}(t)=f(x(t), u(t))
$$

where $x(t) \in \mathbb{R}^{n}$ is the system state vector and $u(t) \in \mathbb{R}^{m}$ the input vector. The vector $x_{e}$ is defined as the equilibrium point if:

$$
\exists x_{e} \in \mathbb{R}^{n} \text {, suchas } f\left(x_{e}(t)\right)=0
$$

with $x(t) \in \mathbb{R}^{n}$ is a constant $\forall t$. In other words, equation (2) needs to converge to the constant $x_{e}$.

\section{Definitions}

The equilibrium point $x_{e}$ is said stable if for every neighborhood $V_{\varepsilon}$ of $x_{e}$, there is a neighborhood $x_{e}$ of $x_{e}$ such that for every initial state $x\left(t_{0}\right)$ of $x(t)$, the $x(t)$ trajectory exists and belongs to $V_{\varepsilon}$ for every $t \geq t_{0}$ :

$$
\forall \varepsilon>0, \exists \alpha>0 \text {, such that } x\left(t_{0}\right)-x_{e}<\alpha, \Rightarrow \forall t>t_{0}, x(t)-x_{e}<\varepsilon
$$

The equilibrium point $V_{\alpha}$ is said attractive if there is a neighborhood $V_{\alpha}$ of $x_{e}$, such that for every initial state $x\left(t_{0}\right)$ of $V_{\alpha}$, the $x(t)$ state trajectory exists and tends to $x_{e}$ values:

$$
\forall t \geq t_{0} \text {, such that } \lim _{t \rightarrow \infty} x(t)=x_{e}
$$

If an equilibrium point $x_{e}$ is attractive and stable, then it is said to be asymptotically stable.

Otherwise, the equilibrium point $x_{e}$ is considered to be unstable [24].

\section{Stability Studies for Linear System}

The equilibrium point $x_{e}$ of a nonlinear system $\lim _{t \rightarrow t_{0}} x(t)=x_{e}$ is said attractive (or stable) if $\lim _{t \rightarrow t_{0}} x(t)=x_{e}$ [24]. In other words, a system is said to be stable if it returns to its steady state after a disturbance by an 
outside element. Notice however that establishing stability conditions for nonlinear systems is an arduous task. Often, a linear approximation of the nonlinear system is employed in order to verify local stability of the nonlinear system around an operating point. In this paper, the kinetic model input is considered as a piecewise constant signal, which amounts to linearizing the system around an operating point.

A linear system (where $A$ is the dynamic matrix), for which the analytical solution is $\left.\left.x(t)=x\left(t_{0}\right) e^{(A(} t-t_{0}\right)\right)$, is considered stable if $A\left(t-t_{0}\right)$ is negative. More explicitly, for a linear system, the real part of the eigenvalues of $A(\Re(\lambda<0$, where $\lambda$ represents the eigenvalues), needs to be negative to ensure stability.

It can be recalled here that finding the eigenvalues of the matrix $A$, with $\operatorname{det}(\lambda I-A)$, is equivalent to finding the roots of the polynomial characteristics $P(\lambda)$ defined by:

$$
p(\lambda)=a_{n} \lambda^{n} a_{n-1} \lambda^{n-1}+\cdots+a_{1} \lambda^{1}+a_{0}
$$

Where $\lambda$ represents the $A$ matrix eigenvalues and $a_{i}$ the polynomial characteristic constant coefficients. The characteristic polynomial degree will be the same as the kinetic model size, i.e., the number of the states. Based on the complexity of the linear system, computation of the roots of the polynomial characteristics can be difficult or impossible. In the following section, an interesting stability test is presented to avoid the explicit computation of the roots.

\section{Routh-Hutwitz Criterion}

In the field of automatic control, the Routh-Hurwitz stability criterion is a mathematical test, which provides a necessary and sufficient condition to characterize the stability of a linear system without computing explicitly the roots of (6). The Routh-Hurwitz test is a recursive algorithm to determine if all the roots of (6) have negative real parts [25]. The Hurwitz Matrix (or Table) helps to conclude on the stability of the linear system: the system is said stable if and only if the determinants of its principal submatrices are all positive [25]. The crux of the Routh-Hurwitz criterion [25] lies in the roots $\lambda$ of the characteristic polynomial of a linear system. These roots with negative real parts represent the stable solutions $e^{(\lambda t)}$ of the system (bounded solutions). The exact calculation of the roots is then replaced by the Routh-Hurwitz criterion whether the roots are positive or negative. Thus, the test provides a means to determine whether the steady state point of a linear system is stable avoiding to directly solving the differential equations that characterize it.

The first two lines of the RH table are filled in column, with the coefficients of the characteristic polynomial (6). The first row contains the coefficients of the terms in $\lambda^{n-2 k}$ ordered by decreasing $n$ power, while the second row contains the coefficients of the terms in $\lambda^{n-1-2 k}$ and ends according to $n$ parity.

Figure 2 illustrates the Routh-Hurwitz table with the polynomial represented by equation (6). The following rows of the Routh-Hurwitz table are built according to the following rules:

$$
b_{n-2}=\frac{-1}{a_{n-1}}\left|\begin{array}{cc}
a_{n} & a_{n-2} \\
a_{n-1} & a_{n-3}
\end{array}\right|, b_{n-i}=\frac{-1}{a_{n-1}}\left|\begin{array}{cc}
a_{n} & a_{n-i} \\
a_{n-1} & a_{n-i-1}
\end{array}\right|
$$

Note that an empty cell is considered equal to zero, if necessary. The calculation of the various cells of the table continues until the first column is completed. The first column of this table is called the pivot column. The Routh-Hurwitz criterion helps concluding whether a system is asymptotically stable when all elements of the pivot column have the same sign. Each change of sign in the elements of the pivot

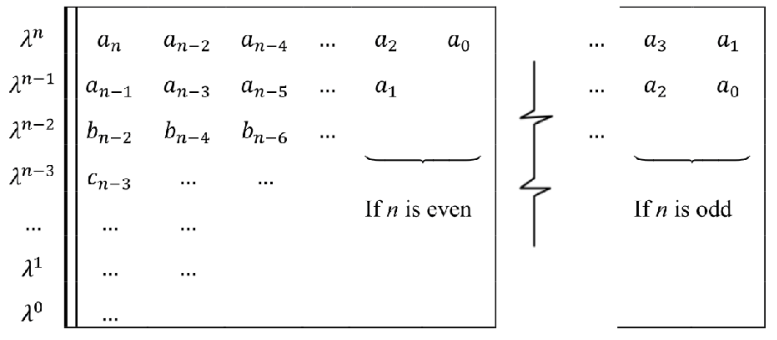

The first column is called the pivots column.

Figure 2: Representation of the Routh-Hurwitz table for a characteristic polynomial of degreen.

column shows the existence of a new positive real part for the roots of the system studied. The appearance of a zero in the pivot column indicates the existence of a pure imaginary root $[25,26]$. In that case, the studied system corresponds to an oscillating system also called pseudo-stable.

The Routh-Hurwitz criterion presented in this section represents a method of determining the location of the roots of a polynomial with constant real coefficients with respect to the left and right halves of the complex plane $\mathbb{C}$ (also called $s$-plane), without actually solving for the roots.

\section{Stability Constraints for a Kinetic Model}

The contribution work, presented in this section, is inspired from $\mathrm{RH}$ criterion to find stability constraints of a linearized kinetic model. To extract constraints guarantying the kinetic model stability, the

\begin{tabular}{|c|c|}
\hline \multicolumn{2}{|c|}{ Algorithm } \\
\hline 1 & $\begin{array}{l}\text { Linearize the system (1) around an operating point considering } \\
\text { the input as a piecewise constant }\end{array}$ \\
\hline 2 & Compute the characteristic polynomial (6) of the linearized system \\
\hline 3 & Construct the RH table with equation (6) and rules (7) \\
\hline 4 & $\begin{array}{l}\text { The first column (pivot column) of the table contains the } \\
\text { constraints needed to guarantee the model stability }\end{array}$ \\
\hline
\end{tabular}
following algorithm is proposed:

The kinetic model will be stable if all constraints provided by the $\mathrm{RH}$ criterion have the same sign (positive or negative). Remark that the constraints will provide only a local stability (around the chosen operating point) due to the linearization. The following examples illustrate the proposed algorithm for designing stability constraints.

\section{Textbook example}

To illustrate the $\mathrm{RH}$ criterion for finding stability constraints on kinetic model parameters, a very basic kinetic model as shown in Figure 3 is used. This kinetic scheme represents a textbook case composed of three states $\left(x_{1}, x_{2}\right.$ and $\left.x_{3}\right)$, four parameters $\left(k_{1}, k_{2}, k_{3}\right.$ and $\left.k_{4}\right)$ and one input $(u)$. This simplified model is represented by a set of ODE, which can be written as $[27,28]$ :

$$
\begin{gathered}
\dot{x}_{1}=k_{2} x_{2}(t)-k_{1} x_{1}(t) u(t) \\
\dot{x}_{2}=k_{1} x_{1}(t) u(t)+k_{4} x_{3}(t)-\left(k_{2}+k_{3}\right) x_{2}(t) \\
\dot{x}_{3}=k_{3} x_{2}(t)+k_{4} x_{3}(t) \\
\text { with } k_{1}=0.7, k_{2}=0.2 \text { and } k_{3}=0.4
\end{gathered}
$$




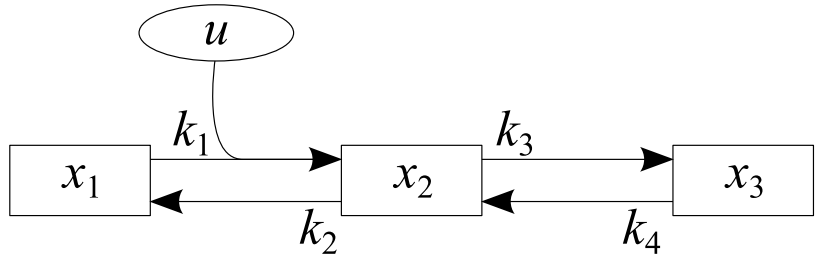

$x_{1}, x_{2}$ and $x_{3}$ represent the model states and $u$ an input to the system. The $k$ parameters represent transition probabilities between the different states.

Figure 3: Schematic representation of a simplified kinetic bilinear model.

\begin{tabular}{|c|c|c|}
\hline$\lambda^{3}$ & $a_{3}$ & $a_{1}$ \\
\hline$\lambda^{2}$ & $a_{2}$ & $a_{0}$ \\
\hline$\lambda^{1}$ & $b_{1}$ & $b_{0}$ \\
\hline$\lambda^{0}$ & $c_{0}$ & \\
\hline
\end{tabular}

Table 1: The Routh-Hurwitz table of the simplified kinetic bilinear model with three states. The first column is the pivots column.

The bilinearity of this model is given by the term $k_{1} x_{1}(t) u(t)$. However, by considering the input $u(t)=u_{0}$ of our simplified model as a piecewise constant, its equation becomes linear:

$$
\begin{aligned}
\dot{x}(t) & =A x(t) \\
\text { with } A & =\left[\begin{array}{ccc}
-k_{1} u_{0} & k_{2} & 0 \\
k_{1} u_{0} & -\left(k_{2}+k_{3}\right) & k_{4} \\
0 & k_{3} & -k_{4}
\end{array}\right]
\end{aligned}
$$

The characteristic polynomial associated with this linear system around an operating point, computed with $\operatorname{det}(\lambda I-A)$, of equation (9) is:

$$
p_{A}(\lambda)=a_{3} \lambda^{3}+a_{2} \lambda^{2}+a_{1} \lambda+a_{0}
$$

The Routh-Hurwitz table of the simplified model is built with the $a_{n}$ parameters of equation (10) and is presented in Table 1 with $a_{3}=1, a_{2}=k_{1} u_{0}+k_{2}+k_{3}+k_{4}, a_{1}=k_{1} u_{0}\left(k_{3}+k_{4}\right)+k_{2} k_{4}, a_{0}=0$, $b_{1}=-\frac{1}{a_{2}}\left(a_{3} a_{0}-a_{2} a_{1}\right)=a_{1}, b_{0}=0$ and $c_{0}=-\frac{1}{b_{1}}\left(a_{2} b_{0}-b_{1} a_{0}\right)=0$.

With a zero $\left(c_{0}\right)$ on the last line of the pivot column, the simple kinetic model used in this example has a purely imaginary root. With a purely imaginary root, this textbook model becomes a particular case from a stability perspective as the stability depends solely on the nature of the input signal. To ensure the stability of the three-state model around the operating point defined by the value of the input $u_{0}$, all parameters of the pivot column must have the same sign. In terms of constraints (cst), this amount to defining the following inequalities:

$$
\begin{aligned}
& \operatorname{cst} 1: 0<1 \\
& \text { cst } 3: 0<k_{1} u_{0}\left(k_{3}+k_{4}\right)+k_{2} k_{4} \\
& \text { cst } 3: 0<k_{1} u_{0}\left(k_{3}+k_{4}\right)+k_{2} k_{4}
\end{aligned}
$$

The purely imaginary root of this textbook example, represented by $c_{0}$ does not provide an asymptotic stability for this model (see Section 2). Only a simple stability can be guaranteed for the three-state system. For example by choosing the parameters $k_{1}=0.7, k_{2}=0.2, k_{3}=0.4$ and $k_{4}=0.4 u_{0}=1.0$ [28], the constraints necessary to have a stable model are met and a simulation around this operating point is shown in Figure 4. An input $u_{0}=1.0$ is applied at $50 \mathrm{msec}$ during $20 \mathrm{msec}$, and is shown in Figure 4A (black). During this interval (50-70 msec), the states respectively presented in Figure $4 \mathrm{~B}, 4 \mathrm{C}$ and $4 \mathrm{D}$ (red, green and blue) change the value of concentration without generating divergence. Once the application of the input $u_{0}$ is interrupted, each state returns to its respective steady state.

It should be noted that some models might receive negative input signals, such as voltage-dependent channels. For this reason, the three-state model was also tested while retaining $u_{0}$ parameters and applying a negative input value equal to -0.5 . With this value of $u_{0}$ it is easy to see that the stability constraints specified by equations (12) and (13) are not met; therefore the model is unstable for a parameter $u_{0}=-0.5$. The instability of the model as provided by the Routh-Hurwitz criterion is verified by the simulation presented in Figure 5. Indeed,

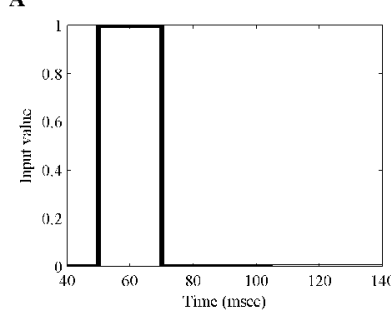

C

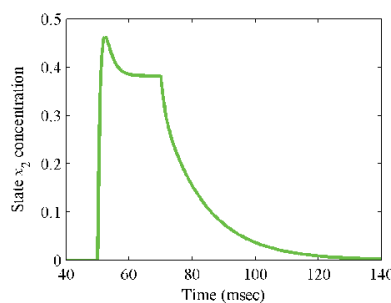

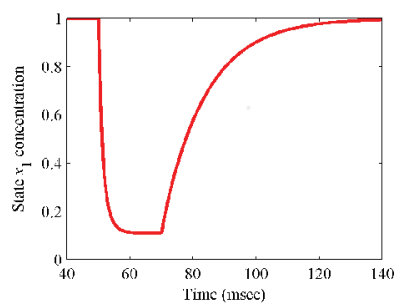

D

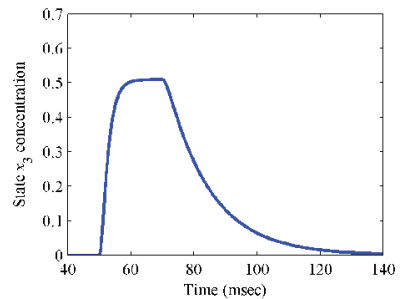

A: input of the system. B: state $x_{1}$. C: state $x_{2} \mathrm{D}$ : state $x_{3}$. All states are stable. Figure 4: Simulation of the simplified model, using parameter values that satisfy stability constraints.
A

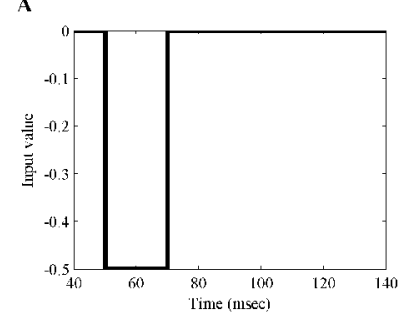

C

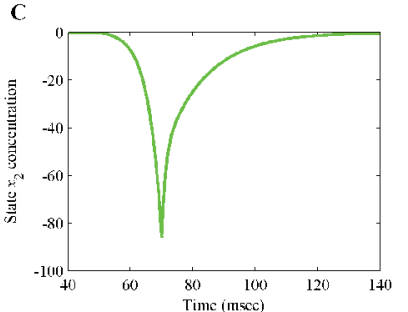

B

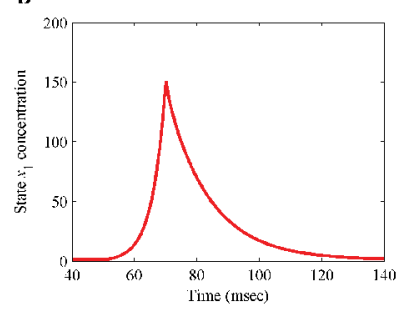

D

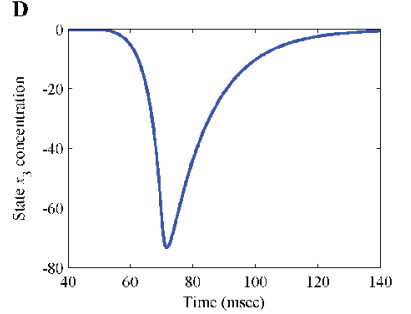

A: input of the system. B: state $x_{1}$. C: state $x_{2}$ D: state $x_{3}$. States $x_{2}$ and $x_{3}$ are unstable as there are negative.

Figure 5: Simulation of the simplified model, using parameter values that do not satisfy stability constraints. 
the concentrations of the various states $\left(x_{1}, x_{2}\right.$ and $\left.x_{3}\right)$ diverge during the application of the input. From a biological point of view, a negative concentration as shown in Figure 5C and 5D of the system states is not possible [28].

When the system is no longer excited by the negative value of $u_{0}$, each state returns to its respective steady state.

The stability constraints (equations (11) to (13)) provide a significant advantage for parameter optimization. Indeed, the constraints provided by the $\mathrm{RH}$ criterion allow determining the range of validity of a varying parameter. It is therefore possible to determine an area of stability in the studied neighborhood. In the case of the linearized three-state model (equation (9)), the validity domain of the parameter $u_{0}$ ensuring stability of the system which depends on other parameters can be obtained by replacing the ...... parameters values in equations (11) to (13). Easily, it may conclude that to ensure the stability of this model, the parameter $u_{0}$ must satisfy the following inequalities:

$$
u_{0}>-k_{2} k_{3} \Rightarrow u_{0}>-0.06
$$

Figure 6 shows the four constraints provided by the RouthHurwitz criterion with the $k_{i}$ parameters previously selected. The dashed line represents the value of $u_{0}=-0.6$. All constraints are positive for all values above this mark (dashed line), whereas below this value some constraints become negative. Thus, stability conditions for the

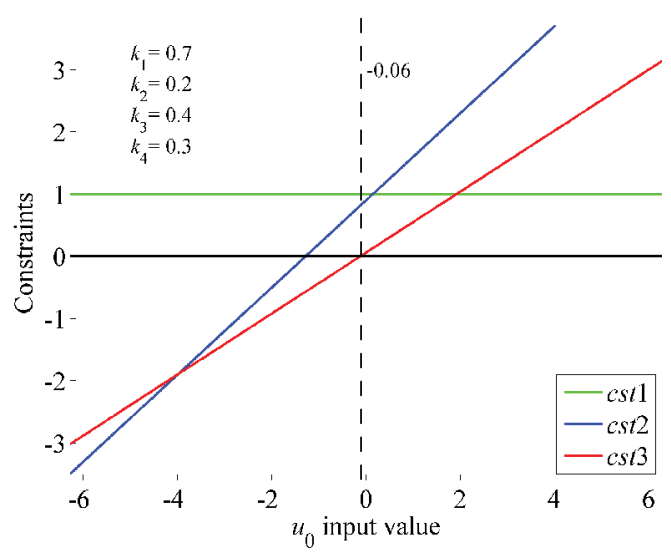

Figure 6: Graphical representation of the four constraints provided by the Routh-Hurwitz criterion for the three-state model.

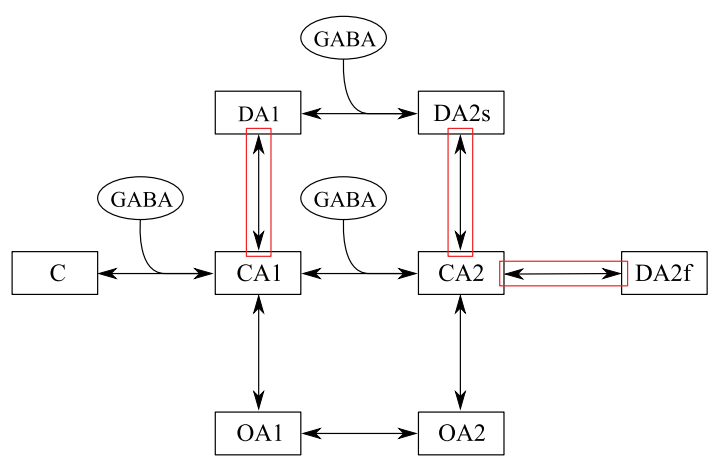

Optimization is considered on association and dissociation parameters outlined in red.

Figure 7: Kinetic scheme of the $\mathrm{GABA}_{\mathrm{A}}$ receptor. model studied around the operating point of interest to the user are determined.

\section{GABA receptor model}

After this textbook case example, the proposed algorithm is applied to a GABA receptor model. The GABA receptor is an ionic receptor and is activated by application of gamma-aminobutyric acid, aka GABA. This ionotropic receptor is of great importance since it binds the main inhibitory neurotransmitter in the CNS and constitutes an important target in the treatment of many CNS diseases $[29,30]$. Figure 7 shows the kinetic scheme of the GABA receptor model developed [30] and used for this study. This model consists of eight states represented by rectangles on the kinetic scheme. A parameter optimization phase is considered to modify the GABA receptor model deactivation to perform the stability analysis. In other word, optimization of association and dissociation parameters outlined in red in Figure 7 will be conducted to determine stability constraints according to these parameters.

Considering the GABA input as a piecewise constant $G A B A_{0}$, it is possible to linearize the system and therefore obtain the following matrix:

$$
A=\left(\begin{array}{cccccccc}
-k a_{1} G A B A_{0} & k d_{1} & 0 & 0 & 0 & 0 & 0 & 0 \\
k a_{1} G A B A_{0} & K_{1} & k d_{2} & 0 & k d_{3} & 0 & k d_{6} & 0 \\
0 & k a_{2} G A B A_{0} & K_{2} & k d_{8} & 0 & k d_{5} & 0 & k d_{7} \\
0 & 0 & k a_{8} & -k d_{8} & 0 & 0 & 0 & 0 \\
0 & k a_{3} & 0 & 0 & K_{3} & k d h_{10} & 0 & 0 \\
0 & 0 & k a_{5} & 0 & k a_{10} G A B A_{0} & K_{4} & 0 & 0 \\
0 & k a_{6} & 0 & 0 & 0 & 0 & -k d_{6} & 0 \\
0 & 0 & k a_{7} & 0 & 0 & 0 & -k d_{7} & 0
\end{array}\right)
$$

with $\quad K_{1}=-\left(k a_{2} G A B A_{0}+k d_{1}+k d_{3}+k d_{6}\right), \quad K_{3}=-\left(k d_{3}+k a_{10} G A B A_{0}\right)$, $K_{3}=-\left(k d_{3}+k a_{10} G A B A_{0}\right)$ and $k_{4}=-\left(k d_{5}+k d_{10}\right)$. Where $k d_{i}$ and $k d_{i}$ represent the association and dissociation rate constants, respectively between the different states of the kinetic scheme of Figure 7 . Computing the determinant of Matrix (14) gives the following characteristic polynomial for the GABA receptor model:

$$
P_{G A B A_{0}}=\lambda^{8}+a_{7} \lambda^{7}+a_{6} \lambda^{6}+a_{5} \lambda^{5}+a_{4} \lambda^{4}+a_{3} \lambda^{3}+a_{2} \lambda^{2}+a_{1} \lambda^{1}+a_{0}
$$

By arranging the parameters of (15) in Table 2 and by applying the Routh-Hurwitz criterion on this table, the constraints that ensure the stability of the model in the first column of this table (pivots column) are obtained.

The stability domain of the $\mathrm{GABA}_{\mathrm{A}}$ receptor model is defined by the pivot column of the Routh-Hurwiz table (Table 2). The parameters in this column represent the constraints to be respected to guarantee the stability of the GABA receptor model during the parameter optimization phase. The parameter $a_{8}$ is equal to 1 , implying that all constraints must be positive to ensure stability of the equilibrium point of the model. However, $\mathcal{D}_{s}$ parameter is equal to zero; which implies that an asymptotic stability cannot be provided. Only stability around the operating point may be guaranteed for this model.

To achieve a deactivation optimization on $\mathrm{GABA}_{\mathrm{A}}$ receptor model $k a_{3}, k d_{3}, k a_{5}, k a_{8}$ and $k d_{8}$ parameter values need to be modified. They represent the optimization settings for our stability study. Using the parameter values given by ref. [31], the stability constraints provided by the Routh-Hurwitz criterion are met and the response of the simulation remains stable for $1 \mathrm{mM}$ of GABA during $250 \mathrm{msec}$ as 


\begin{tabular}{|l|l|l|l|l|l|}
\hline$\lambda^{8}$ & $a_{8}$ & $a_{6}$ & $a_{4}$ & $a_{2}$ & $a_{0}$ \\
\hline$\lambda^{7}$ & $a_{7}$ & $a_{5}$ & $a_{3}$ & $a_{1}$ & \\
\hline$\lambda^{6}$ & $b_{6}$ & $b_{4}$ & $b_{2}$ & $b_{0}$ & \\
\hline$\lambda^{5}$ & $c_{5}$ & $C_{3}$ & $c_{1}$ & & \\
\hline$\lambda^{4}$ & $d_{4}$ & $d_{2}$ & $d_{0}$ & & \\
\hline$\lambda^{3}$ & $e_{3}$ & $e_{1}$ & & & \\
\hline$\lambda^{2}$ & $f_{2}$ & $f_{0}$ & & & \\
\hline$\lambda^{1}$ & $g_{1}$ & & & & \\
\hline$\lambda^{0}$ & $h_{0}$ & & & & \\
\hline
\end{tabular}

Table 2: Representation of the Routh-Hurwitz table for the characteristic

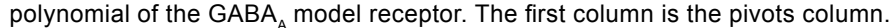

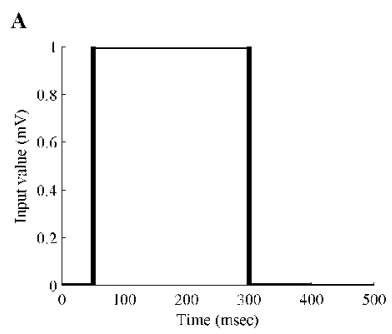

C

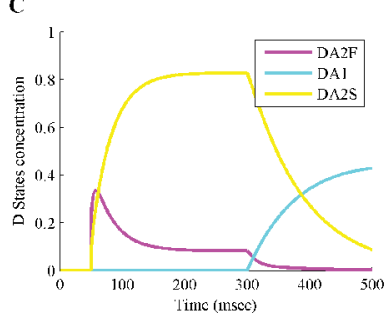

B

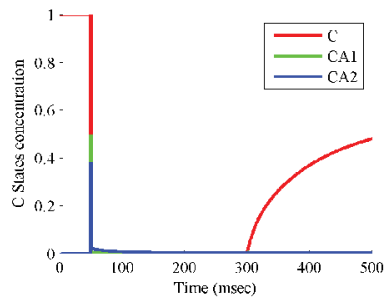

D

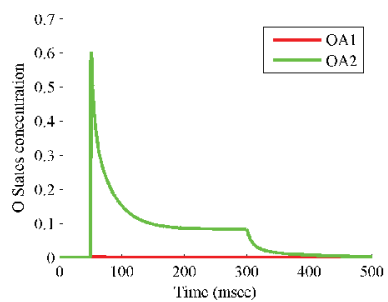

$1 \mathrm{mM}$ GABA is applied as input to the system for $250 \mathrm{msec}$.

Figure 8: Simulation of $\mathrm{GABA}_{A}$ Model receptor with parameters that meet the stability constraints.

shown in Figure 8. The optimization parameters are changed randomly between 0 and +30 . This range of values corresponds to the domain of validity of the association and dissociation rate constants proposed by ref. [31] for our GABA model receptor. This random change in optimization settings places the system in situations where the stability constraints are no longer guaranteed and will generate unstable simulations conditions. Figure 9 illustrates responses of the model in an instable condition, when $1 \mathrm{mM}$ of GABA is applied during $20 \mathrm{msec}$. Instability on the desensitization parameters of the GABA model receptor produces instability on desensitization state 'DA2F'. As shown in Figure 9D, instability on 'OA2' open state appears too, suggesting that the instability propagates in the kinetic model. 'DA2F' state is biologically unstable, as a negative concentration is impossible; 'OA2' state is unstable too: it corresponds to the channel opening probability for the receptor and a probability superior to 1 is therefore impossible ( 1 corresponds to $100 \%$ opening for the receptor).

To perform an analysis of the GABA receptor model, the $\mathrm{RH}$ criterion enables the identification of stability constraints during parameter optimization phase. Indeed, once the model is linearized around the operating point of interest to the user, it is possible to identify constraints that ensure model stability. The constraints may then be integrated within the optimization process making the operation completely transparent to users regardless of their area of expertise.
Besides determining model stability in the parameter optimization phase this criterion may also be used to determine the stability domain of model parameters found in the literature. Indeed, keeping the $\mathrm{GABA}_{\mathrm{A}}$ model receptors with the parameters used in ref. [31], it is possible to determine whether the supplied parameters allow the model to operate in stable conditions. Figure 10 shows the stability constraints of the $\mathrm{GABA}_{\mathrm{A}}$ receptor model with parameters used in ref. [31]. To ensure the stability of the model all constraints must have the same sign. Maintaining the constraint $a_{8}$ to a constant equal to 1 therefore implies that all other constraints must remain positive. Thus, the
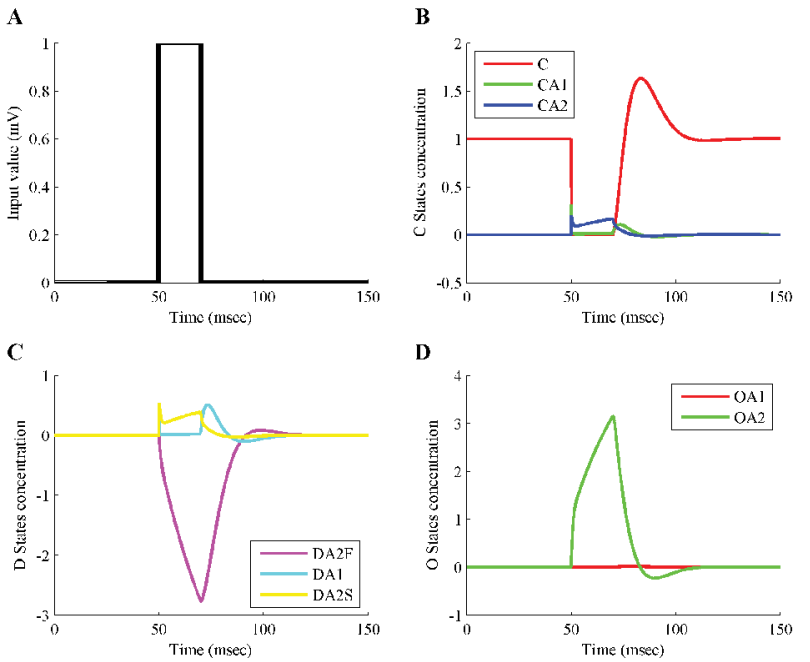

The parameters used were obtained after random change between 0 and 30 of the parameters considered during desensitization optimization. $1 \mathrm{mM}$ GABA is applied as input to the system for $20 \mathrm{msec}$.

Figure 9: Simulation of the $\mathrm{GABA}_{\mathrm{A}}$ receptor model with parameters that do not meet the stability constraints.

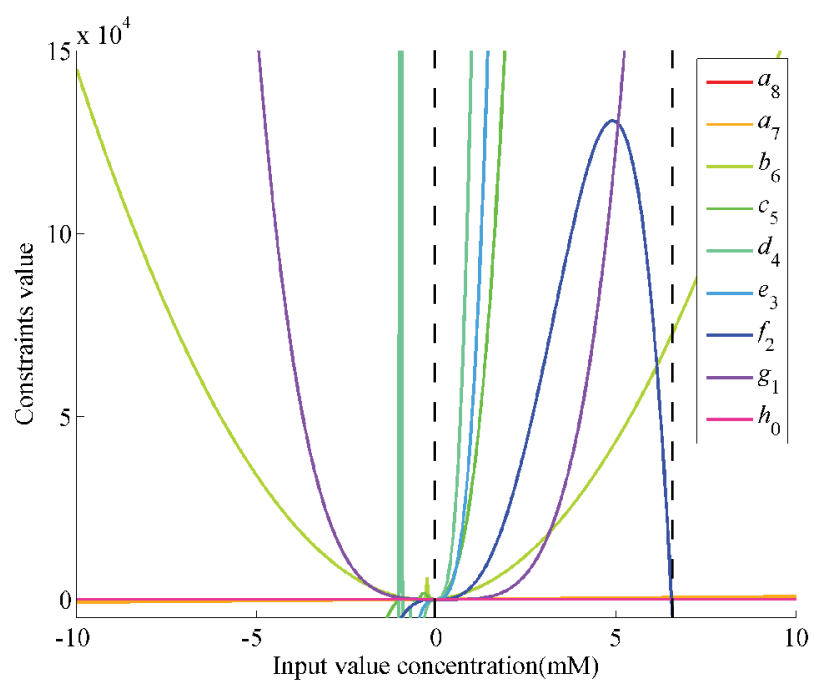

The associations and dissociations parameters are provided from [31]. All constraints are positive or zero between the two red dotted lines, indicating that with [GABA] between 0 and $6.54 \mathrm{mM}$, the $\mathrm{GABA}_{\mathrm{A}}$ receptor model is stable. Figure 10: Graphical representation of stability constraints for $G_{A B A}$ receptor model. 
user knows the values of GABA where the equilibrium point of the linearized model will remain stable.

Figure 10 shows that all constraints are positive or equal to zero between the two dotted lines. Indeed, $h_{0}$ constraint is a constant equal to zero, implying that this $\mathrm{GABA}_{\mathrm{A}}$ receptor model may not be asymptotically stable, but globally stable only if all other constraints are positive. The dotted line on the left corresponds to a GABA concentration equal to $-0.0015 \mathrm{mM}$ while the one on the right corresponds to a GABA concentration equal to $6.54 \mathrm{mM}$. Indeed, for a numerical value of [GABA] less than $-0.0015 \mathrm{mM}$, the constraints $d_{4}, e_{3}$ and $f_{2}$ are negative; whereas for a numerical value of [GABA] greater than $6.54 \mathrm{mM}$ the constraint $f_{2}$ is again negative. It is obvious that no negative GABA concentration may be administered. Finally, the stability domain reading $\mathcal{D}$ of this $\mathrm{GABA}_{\mathrm{A}}$ receptor model with the parameters proposed in ref. [31] is straightforward, as described in Figure 10 . The stability domain $\mathcal{D}$ of our studied bilinear kinetic receptor model can be written as follows:

$$
\mathcal{D}_{s}: G A B A \in[0 ;+6.54]
$$

The Routh-Hurwitz criterion therefore leads us to conclude that for the $\mathrm{GABA}_{\mathrm{A}}$ receptor model with the parameters proposed by Pugh and Raman [31]. The model is globally stable if the concentration of GABA remains between zero and $6.54 \mathrm{mM}$.

\section{Conclusions}

The primary objective of this study was to determine stability constraint to facilitate the development of kinetic models. We proposed to linearize Markov state kinetic models around an operating point considering the input of the bilinear model as piecewise. This linearization allowed application of the Routh-Hurwitz criterion. We first applied this criterion on a textbook case model and demonstrated that the resulting constraints indeed guaranteed stability of the model, thus increasing the chances of success of the optimization phase. Similarly, without the constraints equations guaranteeing the stability, a random parameters change in optimization process may result in instability in the model. In addition to identifying constraints that ensure the stability of synaptic receptor models, the Routh-Hurwitz criterion also allows identification of the stability range, i.e., the determination of the range of input values allowed for a predefined parameter set. We illustrated our approach with a brief study of a $\mathrm{GABA}_{\mathrm{A}}$ receptor model and showed that the parameters provided by Pugh and Raman [31] define a model that is numerically stable if GABA concentration remains under $6.54 \mathrm{mM}$.

It is important to remember that bilinear kinetic receptors are modeled by ODE. The resolution of an ODE is discretized; this implies that bilinear kinetic receptor models are discrete time systems. As the studied models are continuous but resolved by a discrete time method then the stability constraints needs to be obtained with a discrete time criterion. The Jury criterion [32] is the equivalent of $\mathrm{RH}$ criterion for linear discrete time systems. Remark that bilinear kinetic receptor models are mostly hybrid [33]. Hybrid systems combine several operation modes. Voltage-dependent calcium channels (VDCC) are an example of a well-known hybrid system in neuroscience [34]. As presented by Jaffe et al. [34], the equations describing the VDCC model generate a specific behavior for a membrane potential that is negative or equal to zero and a completely different behavior when the potential becomes strictly positive. Future work will consist in adapting the RH and Jury criteria methods to such bilinear hybrid systems. Notice that computational neuroscience often involves models of ever increasing computational complexity [35], our team has already invested significant efforts in reduction of model complexity. Another orientation work may be considered, in parallel to the studied stability constraints here, in investigating a reduction of model order [36,37], while guaranteeing its stability and maintaining desired nonlinear dynamic response.

\section{Conflict of Interest}

The authors declare that they have no competing interests.

\section{Author's Contributions}

MS and RO contributed in design and implementation of the algorithm. NA and AL contributed to develop the kinetic models. All authors contributed equally to writing this paper. All authors read and approved the final manuscript.

\section{Acknowledgements}

Rhenovia Pharma obtained financing from the French National Agency of Research and Technology (ANRT) with a CIFRE scholarship (432/2010). We would like to thank the operational team of Rhenovia Pharma and the MIPS laboratory team (in particular Rachid ATTIA for constructive discussion) who helped for the elaboration of this study.

\section{References}

1. Kitano H (2002) Systems biology: a brief overview. Science 295: 1662-1664

2. Kitano H, Funahashi A, Matsuoka $Y$, Oda K (2005) Using process diagrams for the graphical representation of biological networks. Nat Biotechnol 23 961-966.

3. Hucka M, Finney A, Sauro HM, Bolouri H, Doyle JC, et al. (2003) The systems biology markup language (SBML): a medium for representation and exchange of biochemical network models. Bioinformatics 19: 524-531.

4. Bouteiller JM, Baudry M, Allam SL, Greget RJ, Bischoff S, et al. (2008) Modeling glutamatergic synapses: insights into mechanisms regulating synaptic efficacy. J Integr Neurosci 7: 185-197.

5. Greget R, Pernot F, Bouteiller JM, Ghaderi V, Allam S, et al. (2011) Simulation of postsynaptic glutamate receptors reveals critical features of glutamatergic transmission. PLoS One 6: e28380.

6. Kubinyi H (1977) Quantitative structure--activity relationships. 7. The bilinear model, a new model for nonlinear dependence of biological activity on hydrophobic character. J Med Chem 20: 625-629.

7. Williamson D (1977) Observation of bilinear systems with application to biological control. Automatica 13: 243-254.

8. Zhang L, Zhuge J, Wang Y, Huang R, Liu C, et al. (2009) New insights into oxide traps characterization in gate-all-around nanowire transistors with $\mathrm{TiN}$ metal gates based on combined Ig-Id RTS technique. pp: 46-47.

9. Green DE, Hatefi $Y$ (1961) The mitochondrion and biochemical machines Science 133: 13-19.

10. Eigen M, Hammes GG (1963) Elementary steps in enzyme reactions (as studied by relaxation spectrometry). Adv Enzymol Relat Areas Mol Biol 25 1-38.

11. Fried J (1968) Compartmental Analysis of Kinetic Processes in Multicellular Systems: A Necessary Condition. Phys Med Biol 13: 31-43

12. Mohler RR (1973) Bilinear Control Processes with Applications to Engineering Ecology and Medicine. New York. Academic Press, Orlando, FL, USA.

13. Aganovic Z, Gajic Z (1995) Linear Optimal Control of Bilinear Systems: With Applications to Singular Perturbations and Weak Coupling. Springer Berlin Heidelberg, Berlin New York, USA.

14. Elliott DL (2009) Bilinear Control Systems Matrices in Action. Dordrecht Springer (Netherlands), New York, USA

15. Chen LK, Yang X, Mohler RR (1991) Stability analysis of bilinear systems IEEE Trans Autom Control 36: 1310-1315. 
Citation: Sarmis M, Orjuela R, Bouteiller JC, Ambert N, Legendre A, et al. (2015) Stability Constraints of Markov State Kinetic Models Based on Routh-Hurwitz Criterion. J Comput Sci Syst Biol 8: 296-303. doi:10.4172/jcsb.1000204

16. Luesink R, Nijmeijer H (1989) On the stabilization of bilinear systems via constant feedback. Linear Algebra Its Appl 122-124: 457-474.

17. Lu G, Ho DWC (2006) Continuous stabilization controllers for singular bilinear systems: The state feedback case. Automatica 42: 309-314.

18. Spinu V, Athanasopoulos N, Lazar M, Bitsoris G (2012) Stabilization of Bilinear Power Converters by Affine State Feedback Under Input and State Constraints. IEEE Trans Circuits Syst II Express Briefs 59: 520-524.

19. Florchinger $P$ (1995) Lyapunov-Like Techniques for Stochastic Stability. SIAM J Control Optim 33: 1151-1169.

20. Jerbi $H$ (2001) Global feedback stabilization of new class of bilinear systems. Syst Control Lett 42: 313-320.

21. Lyapunov AM (1904) Problème Général de la Stabilité Du Mouvement. Princeton University Press, USA.

22. Gauthier JP, Bornard G (1980) Stabilisation of bilinear systems, performance specification and optimality. Analysis and Optimization of Systems 28: 125-140.

23. Lu H-C, Wey C-A (1993) Lyapunov stability of bilinear time-delay systems. In Proceedings of the 32nd IEEE Conference on Decision and Control 3841-3842.

24. Khalil HK. Nonlinear Systems. 3rd edn. Prentice Hall, 2001.

25. Routh EJ (1877) A Treatise on the Stability of a given State of Motion, Particularly Steady Motion. University of Michigan Library, USA.

26. Hurwitz A (1964) On The Conditions Under Which an Equation Has Only Roots With Negative Real Parts.

27. Engelberg S (2015) A Mathematical Introduction to Control Theory. 2nd (Rev edn), Imperial College Press, Israel.
28. Dayan P, Abbott LF (2001) Theoretical Neuroscience Computational and Mathematical Modeling of Neural Systems. Massachusetts Institute of Technology Press, USA.

29. Whiting PJ (2003) GABA-A receptor subtypes in the brain: a paradigm for CNS drug discovery? Drug Discov Today 8: 445-450.

30. Brickley SG, Mody I (2012) Extrasynaptic GABA(A) receptors: their function in the CNS and implications for disease. Neuron 73: 23-34.

31. Pugh JR, Raman IM (2005) GABAA receptor kinetics in the cerebellar nuclei: evidence for detection of transmitter from distant release sites. Biophys $\mathrm{J} 88$ 1740-1754.

32. Jury EI (1962) A Simplified Stability Criterion for Linear Discrete Systems. Proc IRE 50: 1493-1500.

33. Zaytoon J (2001) Systèmes dynamiques hybrides. Hermès science publications, Paris.

34. Jaffe DB, Ross WN, Lisman JE, Lasser-Ross N, Miyakawa H, et al. (1994) A model for dendritic $\mathrm{Ca}_{2}^{+}$accumulation in hippocampal pyramidal neurons based on fluorescence imaging measurements. J Neurophysiol 71: 1065-1077.

35. Sarmis M, Bouteiller JMC, Ambert N, Legendre A, Bischoff S, et al. (2013) Assessing Numerical Resolution Methods Performance for Kinetic Models of Receptors and Channels. J Comput Sci Syst Biol 6: 150-164.

36. Guillet J, Mourllion B, Birouche A, Basset M (2011) Extracting second-order structures from single-input state-space models: Application to model order reduction. Int J Appl Math Comput Sci 21: 509-519.

37. Birouche A, Mourllion B, Basset M (2011) Model Reduction for Discrete-Time Switched Linear Time-Delay Systems Via The H? Robust Stability. Control Intell Syst 43: 1753-1763. 